\title{
Interference Subtraction with Supplementary Cooperation in Wireless Cooperative Networks
}

\author{
Zhengguo Sheng*, Zhiguo Ding †, and Kin K Leung* \\ *Department of Electrical and Electronic Engineering, Imperial College, UK \\ $\dagger$ Department of Communication Systems, Lancaster University, UK \\ Email: zhengguo.sheng06@imperial.ac.uk
}

\begin{abstract}
In wireless networks, the broadcast nature of wireless transmission enables cooperation by sharing the same transmissions with nearby receivers and thus can help improve spatial reuse and boost network throughput along a multi-hop routing. The performance of wireless networks can be further improved if prior information available at the receivers can be utilized to achieve perfect interference subtraction. In this paper, we investigate performance gain on network throughput for wireless cooperative networks by using a simple MUD scheme, called overlapped transmission, in which multiple transmissions are allowed only when the information in the interfering signal is known at the receiver. It is shown that the scheme of cooperative transmission with overlapping increases network throughput by $24 \%$ compared to that of direct transmission with overlapping. We then propose a new cooperation scheme called supplementary cooperation, which improves the performance gain of direct transmission with overlapping by $42 \%$. Analytical results are developed to show that in a general network scenario, supplementary cooperation achieves bit error rate (BER) reduction of $34.87 \%$, compared with the conventional cooperative transmission. Furthermore, we proposed a criterion for finding the best cooperative route to achieve maximum network throughput in a general network.
\end{abstract}

\section{INTRODUCTION}

Cooperative transmission (CT) has recently attracted much attention as an effective technique to combat multi-path fading and enhance receiver reliability in wireless communication systems [1]-[5]. The key feature of cooperative transmission is to encourage single-antenna devices to share their antennas cooperatively such that a virtual antenna array can be constructed, thereby, enabling performance gains to be significantly boosted. However, existing work more focuses on reception reliability (i.e., BER) and energy issues; the potential of network throughput of cooperative networks has not been fully explored.

The idea of employing multiuser detection (MUD) in wireless networks to increase spatial reuse and throughput has been proposed in [6]-[9]. Motivated by the fact that prior information available at the receiver can be utilized to achieve

This research was sponsored by US Army Research laboratory and the UK Ministry of Defence and was accomplished under Agreement Number W911NF-06-3-0001. The views and conclusions contained in this document are those of the authors and should not be interpreted as representing the official policies, either expressed or implied, of the US Army Research Laboratory, the U.S. Government, the UK Ministry of Defense, or the UK Government. The US and UK Governments are authorized to reproduce and distribute reprints for Government purposes notwithstanding any copyright notation hereon. perfect interference subtraction by using the MUD scheme [9] and therefore invite more simultaneous transmissions along a multi-hop routing, we propose here to further exploit network throughput in cooperative networks by combining the MUD scheme with supplementary cooperation strategy.

Consider in the two-hop linear cooperative network illustrated in Figure 2, each node acts two roles in the network transmission: source node and relay node. Relay transmission is a main feature of cooperative communication. A one-hop cooperative link between source $A$ and node $C$ includes two different transmission channels: the direct transmission channel, which links source $A$ directly to node $C$ and the relay transmission channel, which links source $A$ through relay $B$ to node $C$. The communication is divided into two orthogonal time slots in order to support the cooperation. During the first time slot, source $A$ broadcasts its frame, which contains data for two destinations $B$ and $C$, and each destination receives its signal. In the second time slot, source $A$ does not transmit, and relay $B$ decodes and forwards the data flow to node $C$. As a result, node $C$ receives two independent copies of the same packets transmitted through different wireless channels, from which diversity gain can be achieved by combining the data copies. The process repeats when node $C$ starts to send the combined data in the third time slot to the node $D$ and $E$. It is worth noting that cooperative communication significantly improves reception reliability, which is an important criterion in the measurement of the performance of cooperative transmissions and will be examined in following sections.

The contribution of this paper is three-fold: first, we illustrate the idea of interference subtraction for cooperative networks by using overlapped transmission, and demonstrate that the scheduling scheme of cooperative transmission with overlapping can obtain larger network throughput than the scheme of direct transmission with overlapping. Second, we propose a new cooperation scheme called supplementary cooperation and then developed to show that it can achieve much better performance than other transmission strategies. Third, in order to balance the trade-off between the BER, reuse factor and network throughput, we propose a criterion for finding the best cooperative route to achieve maximum network throughput in a general network.

The reminder of this paper is organized as follows. In Section II, we show the performance gain of conventional cooperative transmission with overlapping as a motivation for 




Fig. 1. Four-hop direct transmission with overlapping in a five-node linear network

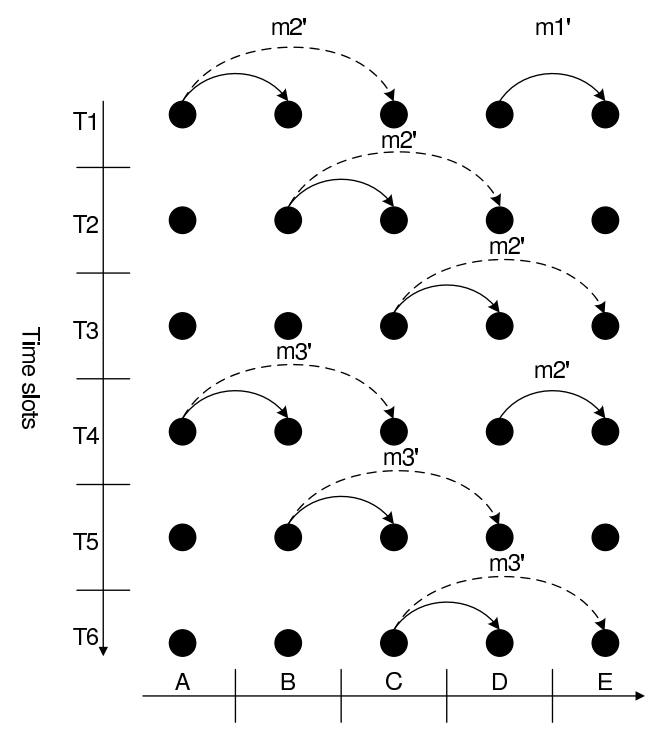

Fig. 2. Two-hop cooperative transmission with overlapping in a five-node linear network

using cooperative transmission. In Section III, we define and analyze the supplementary cooperation. In Section IV, we provide numerical results as well as the criterion and the paper concludes in Section V.

\section{Motivation}

In this section, we illustrate the idea of interference subtraction by using overlapped transmission in a five-node linear network, which is shown in Figure 1 and 2. Without losing generality with previous work [9], the distance between adjacent node is unit one, the transmission range (solid line) is also assumed to be unit one and the interference range (dashed line) is assumed to be twice the transmission range. Specifically, we use outage probability to define the transmission range and interference range.

We employ a propagation model to consider path loss, shadow fading and Rayleigh fading. The wireless link $\mathrm{a}_{i j}$ between the nodes $i$ and $j$ is modeled as $\mathrm{a}_{i j}=h_{i j} / d_{i j}^{k / 2}$, where $d_{i j}$, the distance between the nodes $i$ and $j$, represents the large-scale behavior of the channel gain, $k$ is the path-loss exponent and $h_{i j}$ captures the channel fading characteristics due to the rich scattering environment. In addition, the channel fading parameter $h_{i j}$ is assumed to be independent and identically distributed (i.i.d), complex Gaussian variable with zero mean and unit variance.

For direct transmission, the mutual information between a source and a destination is

$$
I_{D}=\log \left(1+\rho\left|\mathrm{a}_{s, d}\right|^{2}\right),
$$

where $\rho=E_{b} / N_{0}$ is defined as transmission power to noise ratio. Since for Rayleigh fading, $\left|\mathrm{a}_{s, d}\right|^{2}$ is exponentially distributed with parameter $d_{s, d}^{k}$. Thus, the outage probability satisfies

$$
\begin{aligned}
P_{D}^{\text {out }}=\operatorname{Pr}\left[I_{D}<R\right] & =1-\exp \left(-\frac{\left(2^{R}-1\right) d_{s, d}^{k}}{\rho}\right) \\
& =d_{s, d}^{k}\left(\frac{2^{R}-1}{\rho}\right) .
\end{aligned}
$$

where $R$ is the desired data rate in bit/s/Hz which is defined by the quality of service (QoS) requirement and $d$ is the distance between two nodes.

By using (2), we have the equivalent definition as follow

$$
\begin{cases}\text { node is within transmission range, } & \text { if } P_{D}^{\text {out }} \leq \frac{2^{R}-1}{{ }^{\rho}} \\ \text { node is within interference range, } & \text { if } P_{D}^{\text {out }} \leq \frac{2^{k}\left(2^{R}-1\right)}{\rho} \\ \text { interference free, } & \text { if } P_{D}^{\text {out }}>\frac{2^{k}\left(2^{R}-1\right)}{\rho}\end{cases}
$$

Therefore, when setting a desired data rate $R$, a successful transmission can be made only if the outage probability at the receiver satisfies the first condition of (3). When a node is within the interference range, which satisfies the second condition, it cannot directly decode the message from the source. However, from information theory's perspective, it can accumulate the information from both the source and relay to satisfy the first condition by using cooperative transmission in two time slots, which is shown in Figure 2.

For Cooperative transmission, let $d_{s, d}, d_{s, r}$ and $d_{r, d}$ be the respective distances among the source, relay and destination of one single cooperative link. The outage probability is

$$
P_{C}^{\text {out }}=\frac{1}{2} d_{s, d}^{k}\left(d_{s, r}^{k}+d_{r, d}^{k}\right) \frac{\left(2^{R_{C}}-1\right)^{2}}{\rho^{2}} .
$$

Note that the mathematical details behind this equation are omitted due to space limitation and can be found from [10].

As shown in [9], network throughput can be improved by employing simultaneous transmission and the scheduling scheme employing the overlapped transmission for the fivenode linear network is depicted in Figure 1. We observe that in time slot T3, node $C$ forwards packet $m_{2}$, which is received by node $\mathrm{B}$ in $\mathrm{T} 2$, to node $D$. Node $B$ can actually keep a copy of the transmitted message $m_{2}$ locally, thus it knows the message being transmitted by node $C$ in T3 and can apply 
the MUD with the stored prior information $m_{2}$ to mitigate the interference caused by node $C$, while node $\mathrm{A}$ is allowed to transmit another message $m_{3}$ at the same time.

The performance of the scheduling schemes is measured in terms of network throughput at destination $E$. We assume time slots are equal length $T$ and identical transmission power for all nodes. Since destination $E$ successfully receives a message on an average in every two time slots, the average throughput for direct transmission with overlapping is

$$
\lambda_{D}=\frac{R_{D}}{2} .
$$

Under the same BER achievement $P_{C}^{\text {out }}=1-\left(1-P_{D}^{\text {out }}\right)^{2}$, by using (2) and (4), the date rate for cooperative transmission can be increased to

$$
R_{C} \approx R_{D}+\log _{2}\left(\sqrt{\frac{2}{P_{T H}}}\right) .
$$

where $P_{T H}$ is the BER of a two-hop-length direct transmission. For cooperative transmission as shown in Figure 2, a message on an average requires three time slots to be received at destination $E$, the average throughput for cooperative transmission with overlapping is

$$
\lambda_{C}=\frac{R_{C}}{3}=\frac{R_{D}+\log _{2}\left(\sqrt{\frac{2}{P_{T H}}}\right)}{3} .
$$

As a result, if the performance of cooperative transmission with overlapping is better than that of direct transmission with overlapping, only when $\lambda_{D}<\lambda_{C}$, which equals

$$
P_{T H}<\frac{2}{\left(2^{R_{D} / 2}\right)^{2}} .
$$

We observe that (8) can be easily satisfied, especially when the transmission power to noise ratio $\rho$ is large enough. Let us consider an example, we assume $k=2, R_{D}=2 \mathrm{bit} / \mathrm{s} / \mathrm{Hz}$ and $\rho=100$, then with the same BER performance, $R_{C}=$ $3.73 \mathrm{bit} / \mathrm{s} / \mathrm{Hz}$. The transmission efficiency, which is defined as the ratio of network throughput under direct transmission scheduling employing overlapped transmission and cooperative transmission scheduling employing overlapped transmission, is $\Gamma=\frac{\lambda_{C}}{\lambda_{D}}=1.24$.

It is clear that the scheduling scheme of cooperative transmission with overlapped transmission has shown better potential to improve network throughput by $24 \%$ over the scheme of direct transmission with overlapped transmission and that potential can be further improved when implements supplementary cooperation with overlapped transmission as will be examined in the rest of paper.

\section{Supplementary CoOperation}

In this section, we introduce the idea of supplementary cooperation strategy. We have focused so far the conventional cooperation strategy that the mutual information accumulation only happens at the destination node of each cooperative link. Actually, the relay node can also get full benefits from cooperation by taking advantage of the broadcast nature of

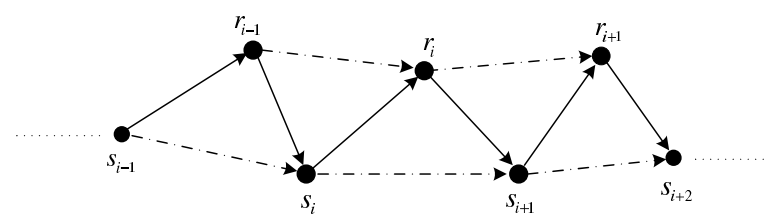

Fig. 3. One example of cooperative route

wireless transmission to further reduce the decoding error. As depicted in Figure 2 that in the second time slot, node $C$ receives the second copy of $m_{2}^{\prime}$ from relay $B$. At the same time, relay $D$ can actually overheard the same $m_{2}^{\prime}$ (dashed line). That is so because the node $D$ is within the interference range of node $B$ and the same packet needs to go through all the nodes along the route. In essence, node $B, C$ and $D$ can compose of another cooperative link called supplementary cooperation.

\section{A. System Model}

Consider a general network scenario where simultaneous transmissions are among the same route which is shown in Figure 3. As an extension, we are interested in the interference impact on network performance, i.e. under a realistic assumption that multiple nodes are active for transmission at the same time. Since we assume that each node uses the same transmission power, the SINR at receiver $s_{i+1}$ is

$$
\rho^{\prime}=\mathrm{SINR}=\frac{P_{t x}}{P_{0}+P_{I}}=\frac{P_{t x}}{P_{0}+\sum_{j} P_{t x}\left|\mathrm{a}_{j, s_{i+1}}\right|^{2}} .
$$

where $P_{I}$ is the summation of interfering power at the receiver. Since for Rayleigh fading, $\left|\mathrm{a}_{j, s_{i+1}}\right|^{2}$ is exponentially distributed with parameter $d_{j, s_{i+1}}^{k}$. By taking the average of $\left|\mathrm{a}_{j, s_{i+1}}\right|^{2}$ and assuming the white noise power $P_{0} \ll P_{I}$, then above SINR is

$$
\rho^{\prime}=\operatorname{SINR} \approx \frac{P_{t x}}{P_{I}}=\frac{\rho}{\sum_{j} \rho / d_{j, s_{i+1}}^{k}}=\frac{1}{\sum_{j} 1 / d_{j, s_{i+1}}^{k}} .
$$

Hence the mutual information at node $s_{i+1}$ can be shown as follow

$$
I=\left\{\begin{array}{l}
\frac{1}{2} \log \left(1+2 \rho_{s_{i+1}}^{\prime}\left|\mathrm{a}_{s_{i}, s_{i+1}}\right|^{2}\right), \\
\text { if }\left|\mathrm{a}_{r_{i-1}, r_{i}}\right|^{2}+\left|\mathrm{a}_{s_{i}, r_{i}}\right|^{2}<G\left(\rho_{r_{i}}^{\prime}\right) \\
\frac{1}{2} \log \left(1+\rho_{s_{i+1}}^{\prime}\left|\mathrm{a}_{s_{i}, s_{i+1}}\right|^{2}+\rho_{s_{i+1}}^{\prime}\left|\mathrm{a}_{r_{i}, s_{i+1}}\right|^{2}\right), \\
\text { if }\left|\mathrm{a}_{r_{i-1}, r_{i}}\right|^{2}+\left|\mathrm{a}_{s_{i}, r_{i}}\right|^{2} \geq G\left(\rho_{r_{i}}^{\prime}\right)
\end{array}\right.
$$

where $\rho_{r_{i}}^{\prime}$ and $\rho_{s_{i+1}}^{\prime}$ are transmission power to interference ratios at relay $r_{i}$ and destination $s_{i+1}$, respectively, and $G\left(\rho_{r_{i}}^{\prime}\right)=\left(2^{2 R}-1\right) / \rho_{r_{i}}^{\prime}$. The first case in (11) corresponds to relay $r_{i}$ that is not being able to decode through supplementary cooperation with $r_{i-1}$ and $s_{i}$, and thus source $s_{i}$ is repeating its transmission. The maximum average mutual information is that of repetition coding from source $s_{i}$ to destination $s_{i+1}$, therefore, the extra factor of 2 is added in the SINR. The second case corresponds to relay $r_{i}$ that has ability to decode and repeat the transmission through supplementary cooperation, then the maximum average mutual information is that repetition coding from both $s_{i}$ and $r_{i}$ to destination $s_{i+1}$. 
Therefore, the outage event for such decode-and-forward is given by $I<R$ and is equivalent to the event

$$
\begin{gathered}
\left(\left\{\left|\mathrm{a}_{r_{i-1}, r_{i}}\right|^{2}+\left|\mathrm{a}_{s_{i}, r_{i}}\right|^{2}<G_{r}\right\} \cap\left\{2\left|a_{s_{i}, s_{i+1}}\right|^{2}<G_{s}\right\}\right) \cup \\
\left(\left\{\left|\mathrm{a}_{r_{i-1}, r_{i}}\right|^{2}+\left|\mathrm{a}_{s_{i}, r_{i}}\right|^{2} \geq G_{r}\right\} \cap\left\{\left|a_{s_{i}, s_{i+1}}\right|^{2}+\left|a_{r_{i}, s_{i+1}}\right|^{2}<G_{s}\right\}\right)
\end{gathered}
$$

where $G_{r}=G\left(\rho_{r_{i}}^{\prime}\right)$ and $G_{s}=G\left(\rho_{s_{i+1}}^{\prime}\right)$. As can be seen, two events of the union in (12) correspond to two cases in (11), respectively. Because the events in union of (12) are mutually exclusive, the outage performance of $s_{i+1}$ with supplementary cooperation (SC) becomes

$$
\begin{gathered}
P_{S C}^{\text {out }}=\operatorname{Pr}[I<R] \\
=\underbrace{\operatorname{Pr}\left[\left|\mathrm{a}_{r_{i-1}, r_{i}}\right|^{2}+\left|\mathrm{a}_{s_{i}, r_{i}}\right|^{2}<G_{r}\right]}_{1} \underbrace{\operatorname{Pr}\left[2\left|\mathrm{a}_{s_{i}, s_{i+1}}\right|^{2}<G_{s}\right]}_{2}+ \\
\underbrace{\operatorname{Pr}\left[\left|\mathrm{a}_{r_{i-1}, r_{i}}\right|^{2}+\left|\mathrm{a}_{s_{i}, r_{i}}\right|^{2} \geq G_{r}\right]}_{3} \underbrace{\operatorname{Pr}\left[\left|\mathrm{a}_{s_{i}, s_{i+1}}\right|^{2}+\left|\mathrm{a}_{r_{i}, s_{i+1}}\right|^{2}<G_{s}\right]}_{4}
\end{gathered}
$$

Here, we compute a closed form for (13). By computing the large SNR behavior, we have the limits $1 \longrightarrow$ $\frac{1}{2} d_{r_{i-1}, r_{i}}^{k} d_{s_{i}, r_{i}}^{k} G^{2}\left(\rho_{r_{i}}^{\prime}\right), 2 \longrightarrow \frac{1}{2} d_{s_{i}, s_{i+1}}^{k} G\left(\rho_{s_{i+1}}^{\prime}\right), 3 \longrightarrow$ $1-\frac{1}{2} d_{r_{i-1}, r_{i}}^{k} d_{s_{i}, r_{i}}^{k} G^{2}\left(\rho_{r_{i}}^{\prime}\right), 4 \longrightarrow \frac{1}{2} d_{s_{i}, s_{i+1}}^{k} d_{r_{i}, s_{i+1}}^{k} G^{2}\left(\rho_{s_{i+1}}^{\prime}\right)$. Then, (13) equals

$$
\begin{aligned}
& P_{S C}^{\text {out }}=\operatorname{Pr}[I<R] \\
& =\frac{1}{4} d_{r_{i-1}, r_{i}}^{k} d_{s_{i}, r_{i}}^{k} d_{s_{i}, s_{i+1}}^{k} G^{2}\left(\rho_{r_{i}}^{\prime}\right) G\left(\rho_{s_{i+1}}^{\prime}\right) \\
& +\frac{1}{2} d_{s_{i}, s_{i+1}}^{k} d_{r_{i}, s_{i+1}}^{k} G^{2}\left(\rho_{s_{i+1}}^{\prime}\right)\left(1-\frac{1}{2} d_{r_{i-1}, r_{i}}^{k} d_{s_{i}, r_{i}}^{k} G^{2}\left(\rho_{r_{i}}^{\prime}\right)\right)
\end{aligned}
$$

where $G\left(\rho_{s_{i+1}}^{\prime}\right)=\left(2^{2 R_{S C}}-1\right) \sum_{j} 1 / d_{j, s_{i+1}}^{k}$ and $G\left(\rho_{r_{i}}^{\prime}\right)=$ $\left(2^{2 R_{S C}}-1\right) \sum_{j} 1 / d_{j, r_{i}}^{k}$. Different to the scenario without interference in (4), the outage probability is no longer a function of transmission power.

\section{B. Transmission Efficiency for Supplementary Cooperation}

Under the same network scenario as depicted in Figure 2, (14) can be simplified as

$$
P_{S C}^{\text {out }}=\frac{d_{s, d}^{k} d_{r, d}^{k}\left(2^{R_{S C}-1}\right)^{2}}{2 \rho^{2}}+\frac{d_{s, d}^{k} d_{s, r}^{k} d_{r^{\prime}, r}^{k}\left(2^{R_{S C}-1}\right)^{3}}{4 \rho^{3}} .
$$

where $d_{r^{\prime}, r}$ is the distance between two adjacent relay nodes. Then we have the date rate

$$
R_{S C}=\log _{2}(x \rho+1) .
$$

where $\quad x=-\frac{b}{3 u}+u-\frac{a}{3}, a=\frac{1}{2^{k-1}}, b=-\frac{1}{3 \cdot 2^{2 k-2}}$

$$
q=-\frac{P_{S C}^{\text {out }}}{2^{2 k-2}}+\frac{2}{27 \cdot 2^{3 k-3}}, u=\sqrt[3]{-\frac{q}{2}-\sqrt{\frac{q^{2}}{4}+\frac{b^{3}}{27}}} .
$$

Consider the same example in Section II, we get $R_{S C}=$ $4.25 \mathrm{bit} / \mathrm{s} / \mathrm{Hz}$ and the average throughput for supplementary cooperation is $\lambda_{S C}=\frac{R_{S C}}{3}$. Then the transmission efficiency of supplementary cooperation employing overlapped transmission with the direct transmission employing overlapped transmission is $\Gamma^{\prime}=\frac{\lambda_{S C}}{\lambda_{D}}=1.42$.

In general, the results from above tell us that the supplementary cooperation with overlapped transmission achieves the best performance among the three schemes. And the gap ratio can be further improved when the SNR increases. It is worth noting that the supplementary cooperation can be realized simply by taking the advantage of the broadcast nature of wireless transmission. Hence, compared with conventional cooperative transmission in Section II, there is no extra system overhead involved.

\section{Numerical Results}

In this section, we evaluate the performance of supplementary cooperation under a general network scenario. Especially, we employ space time reuse scheme to analyze the interference impact on network throughput for later comparison.

We consider here a network scenario that 100 nodes are uniformly distributed in a $1000 \mathrm{~m} \times 1000 \mathrm{~m}$ topology with the source and destination nodes located at the top left corner and the bottom right corner, respectively. We set the transmission power to noise ratio $\rho=60 \mathrm{~dB}$, desired data rate $R=0.2 \mathrm{bit} / \mathrm{s} / \mathrm{Hz}$. Results are averaged over 100 simulation runs. By using the cooperative routing algorithm [11], Figure 4 reports the end-to-end BER performance of routes with different total number of hops from the source to destination. In this simulation, we consider the simplest case where only one transmission is possible in each time slot. So there is no other interference present. As can be seen, the supplementary cooperation achieves much better error performance than the conventional cooperation as well as the same route with only direct transmission. Especially, an average BER reduction of $34.87 \%$ is achieved when compared with the conventional cooperation. In addition, it further shows that for cooperative routing, the end-to-end BER improves as the number of hops in the selected route increases. In particular, we observe that 3 hops supplementary cooperation already has the better end-toend BER performance than 4 hops conventional cooperation. Such implies that supplementary cooperation can generate routes with a smaller number of hops and satisfactory end-toend BER when compared with the conventional cooperation.

Next, we evaluate the end-to-end BER performance of supplementary cooperation with overlapped transmission under an interfering environment which allows multi-node transmissions along the same routing using space time reuse scheme.

We assume all nodes along a route transmit in the same frequency band and employ a regular TDM-schedule of length $\mathrm{M}$-cooperative-links so that in time slot $t$, the nodes $2 i \mathbf{M}+(t \bmod 2 \mathrm{M})$ are allowed to transmit, for $i=\ldots-1,0,1 \ldots$. Because each cooperative link composes of two transmissions from the source and relay in two consecutive time slots, therefore, the extra factor 2 is added in TDM-schedule. Figure 6 shows the TDM-schedule for a general cooperative route with reuse factor $\mathrm{M}=1$. The solid lines are simultaneous 


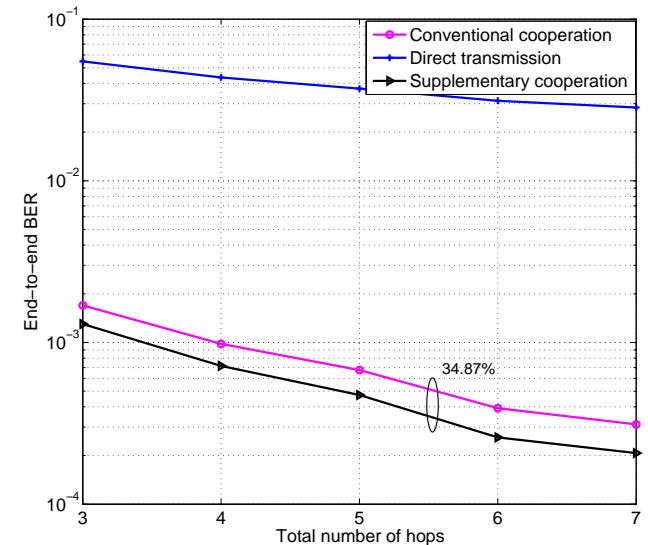

Fig. 4. Bit Error Rate vs. Total Number of Hops

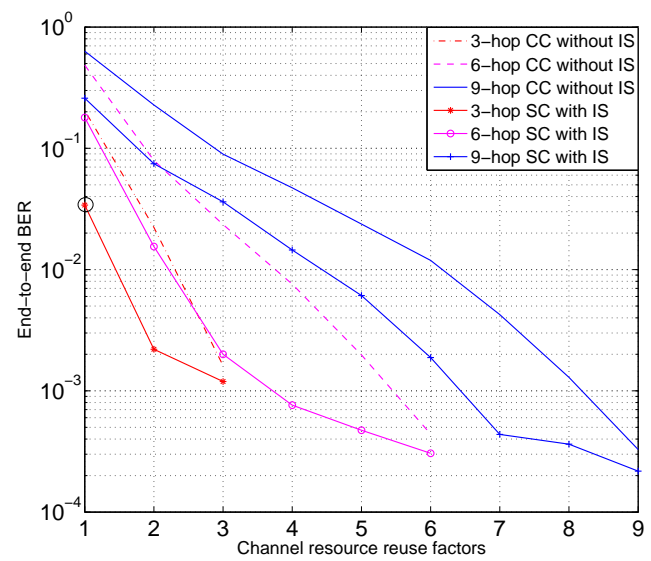

Fig. 5. Bit Error Rate vs. Channel Resource Reuse Factors

transmissions and the dashed lines are interferences that can be canceled through overlapped transmissions.

Figure 5 shows the end-to-end BER performance of supplementary cooperation (SC) with interference subtraction (IS) by using the overlapped transmission and conventional cooperation (CC) without IS. It is clear that supplementary cooperation with interference subtraction achieves much better performance than conventional way. Furthermore, a careful reader might notice that in fact there is a trade-off between reuse factor, end-to-end BER and network throughput. As reuse factor increases, end-to-end BER is reduced correspondingly. However, the network throughput is adversely affected by large reuse factor. Therefore, in order to find the best cooperative route achieving maximum network throughput, we define the network throughput as follow

$$
\lambda= \begin{cases}R\left(1-P_{E T E}\right) /(2 \mathrm{M}+1), & \text { if } \mathbf{M}=1 \\ R\left(1-P_{E T E}\right) /(2 \mathrm{M}), & \text { if } \mathbf{M}>1\end{cases}
$$

where $R$ is the desired data rate, $P_{E T E}$ is the end-to-end BER and $\mathrm{M}$ is the reuse factor. By using (17), we find that 3 hops supplementary cooperation with interference subtraction is the best routing to achieve the maximum throughput in such

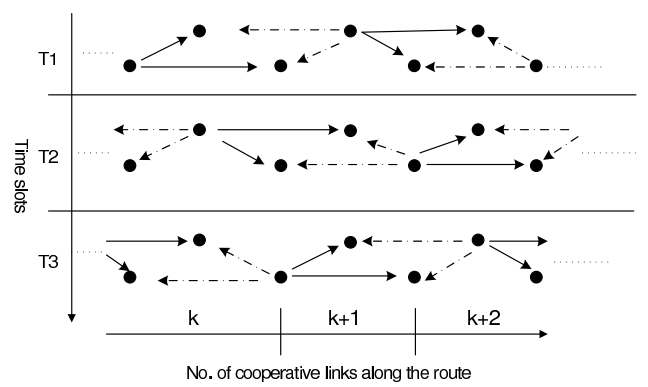

Fig. 6. TDM-schedule for a cooperative route with $M=1$

network scenario, which is circled in Figure 5.

\section{CONCLUSION}

In this paper, we have illustrated the idea of interference subtraction for cooperative networks by using overlapped transmission. Especially, it has shown that the scheme of the conventional cooperative transmission with overlapping obtains larger network throughput than that scheme of direct transmission with overlapping, an improvement of $24 \%$ is achieved. Then, we have proposed a new cooperation scheme called supplementary cooperation, which further improves the network throughput of direct transmission with overlapping by $42 \%$. Analytical results are developed to show that in a general network scenario, compared with the conventional cooperative transmission, the supplementary cooperative transmission achieves the BER reduction by $34.87 \%$. Furthermore, in order to balance the trade-off between reuse factor, BER and network throughput, the proposed criterion can help us find the best cooperative route achieving maximum network throughput in a general network.

\section{REFERENCES}

[1] A. Scaglione, D. Goeckel, and J. N. Laneman, "Cooperative communications in mobile ad-hoc networks: Rethinking the link abstraction," IEEE Signal Processing Magazine, vol. 23, pp. 18-29.

[2] J. N. Laneman and G. W. Wornell, "Distributed space-time-coded protocols for exploiting cooperative diversity in wireless networks," IEEE Trans. Information Theory, vol. 49, pp. 2415-2425, Oct. 2003.

[3] J. N. Laneman, D. N. Tse, and G. W. Wornell, "Cooperative diversity in wireless networks: Efficient protocols and outage behavior," IEEE Trans. On Information Theory, vol. 50(12), Dec. 2004.

[4] A. E. Khandani, "Cooperative routing in wireless networks," Ph.D. dissertation, MIT, 2004.

[5] F. Li, K. Wu, and A. Lippman, "Energy-efficient cooperative routing in multi-hop wireless ad hoc networks," in IEEE International Performance Computing and Communications Conference, 2006.

[6] D. C. S. S. Ghez, S. Verdu, "Stability properties of slotted aloha with multipacket reception capability," IEEE Trans. on Automatic Control, vol. 33, pp. 640-649, 1988.

[7] B. W. M. Howlader, K. Mostofa, "System architecture for implementing multiuser detector within an ad-hoc network," Military Communication Conference, 2001.

[8] H. V. P. C. Comaniciu, N. B. Mandayam, Wireless Networks: Multiuser Detection in Cross-Layer Design. Springer, 2005.

[9] S. Boppana and J. M. Shea, "Overlapped transmission in wireless ad hoc networks," in International Conference on Comuunication, Circuits and Systems, 2006.

[10] Z. Ding and K. K. Leung, "Cross-layer routing optimization for wireless networks with cooperative diversity," IEEE PIMRC, 2008.

[11] Z. Sheng, Z. Ding, and K. K. Leung, "On the design of a quality-ofservice driven routing protocol for wireless cooperative networks," in IEEE Vehicular Technology Conference, Singapore, 2008. 